\title{
Moyra Smith: Translational research in genetics and genomics
}

\author{
Oxford University Press 2008, pp220, £32.99, ISBN 978-0-19-531376-5
}

\author{
Patrick J. Morrison
}

Published online: 13 December 2008

(C) Springer-Verlag 2008

Having had two recent letters from my non-medically qualified chief executive congratulating me on my contributions to 'translational research', I was wondering exactly what his perception of translational research was. I always thought I was doing applied clinical research and in doing so, helping patients in my clinics. This book entitled 'translational research in genetics and genomics' therefore arrived just in time to help me find the answer.

A helpful introductory chapter discusses isolation of genes through genomics-areas I was familiar with in the 1990s such as linkage disequilibrium and haplotyping have taken on a deeper meaning when using Tag SNP's and HapMap to further elucidate diseases such as Type I and II diabetes, age related macular degeneration and other 'polygenic' diseases such as Crohn's and ulcerative colitis. The text is extremely up to date with a large number of 2007 references and I found each short chapter an interesting and informative read. Chapters discussing copy number variation, deletion polymorphisms, array technology, significance of structural chromosomal changes and highresolution cytogenetics are succinct and stimulating. Clinical dysmorphologists may now find it easier just to do the 'blood test' and then surprise their colleagues at the case discussion meeting with the completely unexpected microdeletion or subtle chromosomal rearrangement that has been found.

Older clinical geneticists will find the chapter on the significance of DNA sequence changes for diagnosis and therapy very hopeful. Old genetics textbooks always relegated

P. J. Morrison ( $\square)$

Clinical Genetics, Belfast HSC Trust,

City Hospital Campus, Lisburn Road, Belfast BT9 7AB, UK

e-mail: patrick.morrison@belfasttrust.hscni.net 'therapy' to the concluding sentence of the two-page 'future directions' final chapter obligatory in all such 1990s books. Duchenne muscular dystrophy and cystic fibrosis and therapeutic possibilities for spinal muscular atrophy and other diseases are described and with us now. Those unsure about epigenetics have an excellent chapter explaining all. The chapter on late onset diseases discusses dementia, Parkinson's disease and other conditions and I was pleased to find Huntington disease - a personal interestrefreshingly hardly mentioned, a reflection on progress on diagnosis and management, and the increasing life expectancy throwing out newer and more common and relevant ageing challenges for this century.

Chaperones and gene-based therapies including enzyme replacement therapy find a complete chapter, as does an explanation of mTOR/rapamycin pathways in tuberose sclerosis and neurofibromatosis. I learnt the definition of 'toxigenomics' - 'molecular response to biological agents' allowing fingerprints of exposure to be identified. The book finishes with therapeutic applications in cancer including PARP inhibitors, and a discussion of metastases, and a discussion on where Biobanks have got to.

This is an excellent book for all clinical geneticists wanting to know where progress in genetics is at, and is particularly good for both the trainee and the 'older geneticist' who want to understand where research is going and the new terminology used. It is an excellent brief and understandable guide to recent advances in genetics.

Now I am confident, I know what translational research is - it is the modern name for applied clinical researchand this book nicely covers the gap between scientific discovery and the translation of these discoveries into clinical practice. 